\title{
On the Meaning of the General Factor of Psychopathology ("P-Factor") in Symmetrical Bifactor Models
}

Manuel Heinrich, Christian Geiser, Pavle Zagorscak, G. Leonard Burns, Johannes Bohn, Stephen P. Becker, Michael Eid, Theodore P. Beauchaine \& Christine Knaevelsrud

\begin{abstract}
Author Notes
Manuel Heinrich, Pavle Zagorscak, Johannes Bohn \& Christine Knaevelsrud, Department of Education and Psychology, Division for Clinical Psychological Intervention, Freie Universität Berlin; Christian Geiser, Department of Psychology, Utah State University. G. Leonard Burns, Department of Psychology, Washington State University. Stephen P. Becker, Division of Behavioral Medicine and Clinical Psychology, Cincinnati Children's Hospital Medical Center, and Department of Pediatrics, University of Cincinnati College of Medicine. Michael Eid, Department of Education and Psychology, Division for Methods and Evaluation, Freie Universität Berlin. Theodore P. Beauchaine, Department of Psychology, Ohio State University.

Correspondence concerning this article should be addressed to Manuel Heinrich, Department of Education and Psychology, Division of Clinical-Psychological Intervention, Freie Universität Berlin, Habelschwerdter Allee 45, 14195 Berlin, Germany, e-mail: manuel.heinrich[at]fu-berlin.de
\end{abstract}

Please note: This is a preprint and has not been peer reviewed. 


\begin{abstract}
Symmetrical bifactor models are frequently applied to diverse symptoms of psychopathology to identify a general $P$ factor. This factor is assumed to mark shared liability across psychopathology dimensions and mental disorders. Despite their popularity, however, symmetrical bifactor models often yield anomalous results, including but not limited to nonsignificant or negative specific factor variances and non-significant or negative factor loadings. To date, these anomalies have often been treated as nuisances to be explained away. In this paper, we demonstrate why these anomalies alter the substantive meaning of $P$ such that it (1) does not reflect general liability to psychopathology and (2) differs in meaning across studies. We then describe an alternative modeling framework, the bifactor- $(S-1)$ approach. This approach avoids anomalous results, provides a framework for explaining unexpected findings in published symmetrical bifactor studies, and yields a general factor with well-defined meaning across studies. We present an empirical example to illustrate these points and provide concrete recommendations to help researchers decide for or against a specific variant of bifactor structures. In summary, bifactor- $(S-1)$ models provide an approach to answer questions posed in symmetrical bifactor models in a more comparable and replicable manner.
\end{abstract}

Keywords: $P$ factor, general factor of psychopathology, bifactor, bifactor- $(S-1)$, anomalous results, collapsing factors. 
On the Meaning of the General Factor of Psychopathology ("p-Factor") in Symmetrical Bifactor Models

Many studies use bifactor models to characterize features that different domains of psychopathology (e.g., internalizing, externalizing, thought disorders) have in common (e.g., Carragher et al., 2016; Caspi et al., 2014; Gomez et al., 2019; Haltigan et al., 2018; Laceulle et al., 2015; Lahey et al., 2012, 2018; Markon, 2019; Martel et al., 2017; Swales et al., 2020; Tackett et al., 2013). In bifactor models, psychopathology is structured in terms of (1) a general factor on which all indicators of all domains load and (2) domain-specific factors that depict variance not shared with the general factor. In clinical psychology, the general factor has been referred to as the "general factor of psychopathology," or " $P$-factor" (e.g., Caspi et al., 2014). $P$ if often assumed to be transdiagnostic in the most general sense - in other words, a latent manifestation of a single causal factor associated with every symptom of every mental disorder.

The growing number of bifactor applications suggests that modeling a general factor provides a major advantage over a model with correlated factors. The term "general factor of psychopathology" suggests a clear and substantively meaningful interpretation. Initially identified by Lahey et al. (2012), $P$ is typically interpreted as an overarching vulnerability to psychopathology derived from a common etiological mechanism. Many authors, including Caspi et al. (2014), attribute $P$ to deficiencies in frontally-mediated executive processes, such as inhibitory control and self-/emotion regulation (see, e.g., Beauchaine \& Cicchetti, 2019; Martel et al., 2017). According to such theories, compromised executive and self-regulatory abilities potentiate virtually all forms of psychopathology including internalizing, externalizing, and thought problems through failures in top-down cortical inhibition of subcortical neural systems (see Beauchaine \& Zisner, 2017 and Carver et al., 2017 for related interpretations and see Smith et al., 2020 for alternative conceptualizations of P). 
Although the $P$ factor has generated lively discussions over shared etiological mechanisms of psychopathology, these discussions assume the general factor is a valid representation of shared liability. Recently, evidence has emerged that questions this assumption. It is therefore unclear to what extent the general factor in a bifactor model indeed represents general psychopathology_or whether it measures something different. This ambiguity is one reason why the increasing use of bifactor models has been critically scrutinized by a number of researchers (e.g., Bonifay et al., 2017; Burns et al., 2020a; Eid et al., 2017; Heinrich et al., 2020; Sellbom \& Tellegen, 2019; van Bork et al., 2017; Watts et al., 2019). This scrutiny suggests it is crucial to define conditions under which the general factor can be interpreted in an unambiguous and meaningful manner.

In this manuscript, we argue that commonly applied symmetrical bifactor models

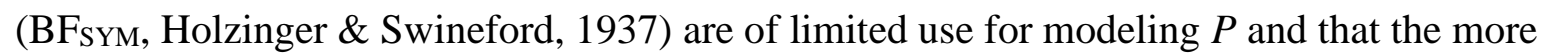
theory-oriented bifactor- $(S-1)$ approach $\left(\mathrm{BF}_{S-1}\right.$, Eid et al., 2017$)$ is preferred. Due to anomalous results, general factors in $\mathrm{BF}_{\mathrm{SYM}}$ models typically do not depict the overarching factor that researchers intend to model, but instead carry meaning that varies from study to study. We explain how the $\mathrm{BF}_{S-1}$ approach addresses shortcomings of $\mathrm{BF}_{\mathrm{SYM}}$ models and provides a general factor with well-defined meaning. Although we agree that general vulnerabilities may be useful explanatory constructs for characterizing the emergence and persistence of various mental disorders, we argue that $\mathrm{BF}_{\mathrm{SYM}}$ models typically do not meaningfully depict these communalities. We illustrate our arguments by means of various published bifactor models. Our aim is not to criticize these studies or authors (indeed, some of us are guilty of the same misinterpretations), but to contribute to a better understanding of the meaning and interpretation of the $P$-factor as depicted in bifactor models.

\section{Symmetrical Bifactor Models}

When thinking about bifactor structures, researchers usually have $\mathrm{BF}_{\mathrm{SYM}}$ models in mind (see Figure 1, Model 2 for an example). In these models, each item or scale loads on a 
general factor - the general factor of psychopathology — and a domain-specific $S$ factor (Eid et al., 2017; Holzinger \& Swineford, 1937). Variance of each observed indicator is decomposed into three parts. The first part is determined by the general factor and must be different from zero. Otherwise, the item has nothing in common with the general factor of psychopathology that is supposed to underlie all symptoms. The second part is variance due to a narrower domain-specific factor. Since each item should also represent a specific domain of psychopathology (e.g., internalization), this part must be meaningfully different from zero as well. The third part comprises measurement error and systematic variance that is unique to an item and not shared with other items.

$S$ factors are residual factors with a mean of zero that capture deviations of the domain-specific values from values expected based on the general factor (Eid et al., 2017; Reise, 2012). Correlations between $S$ factors must be fixed to zero in the BF $\mathrm{SYM}_{\mathrm{SM}}$ approach. This follows from the assumption that the general psychopathology factor causes different domains of psychopathology to correlate. Those correlations should therefore vanish when effects of the general factor are removed (Eid et al., 2017; Reise, 2012). Reise et al. (2012) point out that correlations between $S$ factors contradict the idea of a single unifying factor because they suggest "the presence of additional and unmodeled general factors" (p. 692). Furthermore, the general factor and all $S$ factors are uncorrelated by definition (Eid et al., 2017; Holzinger \& Swineford, 1937).

Among many researchers, $\mathrm{BF}_{\mathrm{SYM}}$ models are considered theoretically or empirically superior to correlated first-order factors models (see Figure 1, Model 1), because they appear to include a single overarching dimension that explains why dimensions of psychopathology

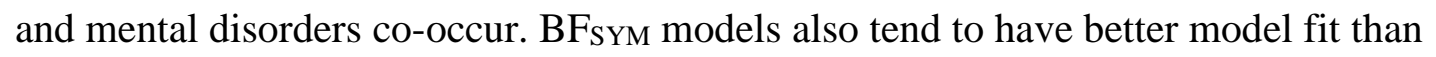
correlated first-order factor models because they estimate additional parameters (Sellbom \& Tellegen, 2019). When applied in practice, however, $\mathrm{BF}_{\mathrm{SYM}}$ models typically yield solutions that contain improper ("inadmissible") parameter estimates (e.g., negative residual variances 
or negative specific factor variances) and/or otherwise anomalous results that are either difficult to interpret or fully uninterpretable (e.g., very small or negative factor loadings). As we describe in detail below, such results challenge the assumption that $P$ represents a general factor that underlies all symptoms, and instead empirically define $P$ as a specific domain of psychopathology (i.e., internalizing, externalizing, or thought disorders), depending on sample-specific solutions.

\section{Anomalous Results in $\boldsymbol{P}$ Factor Studies}

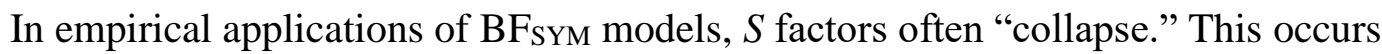
when an $S$ factor has a very small or negative variance estimate and/or when many of the standardized loadings on the $S$ factor in a bifactor model are close to zero (e.g., $\leq .2)$ and/or non-significant. When a factor has no variance and/or when only a few indicators have substantial loadings on that factor, the factor in question may not exist. In this case, indicators that should depict both (1) transdiagnostic vulnerability as expressed by the general factor and (2) a specific domain of psychopathology, instead only measure $P$.

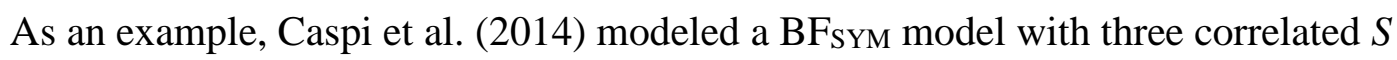
factors (internalizing, externalizing, thought disorder) and reported a negative variance for the indicator assessing obsessive-compulsive disorder (OCD). Caspi et al. (2014) dropped the specific thought disorder factor from their model to deal with this inadmissible estimate. Romer et al. (2019, see also Romer et al. 2018) did the same reporting a Heywood case for the mania indicator after including the general factor in a model that used the same set of $S$ factors as Caspi et al. (2014). Like Caspi et al. (2014), Romer et al. (2019) omitted the specific thought disorder factor. Thus, instead of $P$ being a general factor underlying all 11 symptom dimensions in the study, $P$ became a thought disorder factor (represented by OCD, mania, and schizophrenia) in both studies. We explain this point and elaborate resulting problems with interpretation in detail shortly. 
Lahey et al. (2012, their Figure 1) also examined three domains of psychopathology (distress, fear, externalizing) with a bifactor model. The generalized anxiety disorder (GAD) and agoraphobia/panic (AP) indicators did not load substantially on their respective $S$ factors (standardized loadings: GAD = .13; $\mathrm{AP}=.16$ ), but both indicators loaded strongly on the general factor $(\mathrm{GAD}=.85$ and $\mathrm{AP}=.77)$. As we explain below, these findings indicate that the $P$ factor was in fact a GAD/AP factor — not a general liability factor.

In addition, in some bifactor applications, it is challenging to properly interpret the $S$ factor because patterns of factor loadings differ from those expected based on the model with correlated factors. For example, if all factor loadings in the correlated factor model have the same sign, they should have the same sign even after the general factor is included. Instead, factor loadings often inconsistently change signs (some from positive to negative while others remain positive). Such changes are often problematic because they are unexpected and lack a theoretically sound explanation.

For example, in applying a bifactor model with uncorrelated $S$ factors to fear, distress, and externalizing, Watts et al. (2019) reported a negative loading (-.34) for the general anxiety indicator on its specific distress factor. In contrast, the same indicator had a strong positive loading on $P(.84)$. The only other indicator of the distress specific factor (major depression) loaded moderately (.36) on the same $S$ factor but loaded .77 on the general $P$ factor. In addition, generalized anxiety and major depression were the only indicators that loaded highly on the general factor (all other general factor loadings were $\leq .49$ ). These findings render the specific distress factor difficult to interpret and any interpretation of $P$ as a general factor questionable (here, $P$ was defined primarily by generalized anxiety and major depression indicators, which did not have a stable $S$ factor).

Anomalous results are not specific to bifactor models intended to characterize $P$. In their review of 82 bifactor studies across different areas of psychology, Eid et al. (2017) found anomalous results in at least $50(61 \%)$ of applications. Heinrich et al. (2020) reported that 
similar problems occur frequently in applications of the bifactor approach to research on depression. Burns et al. (2020a) examined 24 bifactor applications of attention deficit/hyperactivity disorder (ADHD) symptoms or ADHD/oppositional defiant disorder (ODD) symptoms, of which over $75 \%$ yielded anomalous loading patterns or inadmissible solutions. Thus, anomalous results are unexceptional and instead represent a general problem

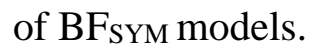

Why Collapsing Factors Change the Meaning of $\boldsymbol{P}$. As described above, a frequent problem in applications of BFSYM models is that one $S$ factor has weak, inconsistent, and/or negative loadings, or a variance estimate that is zero or close to zero. Collapsing specific factors in BFSYM models change indicators of the collapsing factor into "pure" indicators of $P-$ not indicators of a specific factor. As a consequence, the meaning of the general factor is defined completely by specific factor items, and $P$ becomes equivalent to the corresponding first-order factor for the collapsing facet. The "general" $P$-factor is therefore no longer a general factor of psychopathology. Instead, it is an empirically defined latent variable underlying indicators of the collapsed factor. This is similar to an exploratory factor analysis in which items that have no cross-loadings but load strongly on one factor are considered as putative markers of that construct.

The fact that the "general" $P$ factor in models with collapsing specific factors is no longer interpretable as a general factor can be readily seen through the measurement equation in a $\mathrm{BF}_{\mathrm{SYM}}$ model with orthogonal $S$ factors. Let us assume that the model contains a specific thought disorder (TD) factor in addition to a specific internalizing and externalizing factor, and that we use sum scores that measure the severity of mania (MAN), OCD, and psychosis as indicators of the thought disorder factor. The bifactor measurement equation for mania is given by $M A N=\alpha_{\mathrm{MAN}}+\lambda_{\mathrm{P}, \mathrm{MAN}} \times P+\lambda_{\mathrm{S}_{-} \mathrm{TD}, \mathrm{MAN}} \times S_{\mathrm{TD}}+\varepsilon_{\mathrm{MAN}}$. That is, the observed score of mania is the sum of an intercept $\left(\alpha_{\text {MAN }}\right)$, the general factor score weighted with the 
factor loading of mania on $P\left(\lambda_{\mathrm{P}, \mathrm{MAN}}\right)$, the $S$ factor score weighted by the factor loading of mania on the specific thought disorder factor $\left(\lambda_{S_{-} \text {TD,MAN }}\right)$, and measurement error $\left(\varepsilon_{\text {MAN }}\right)$.

When $S_{\mathrm{TD}}=0$ (i.e., the variance of the $S$ factor is zero or non-significant/very small), $\lambda_{S_{-} \text {TD,MAN }}=0$ (i.e., the factor loading of mania on the specific thought disorder factor is fixed to zero or non-significant/very small), or when $\lambda_{\mathrm{S}_{-} \mathrm{TD}, \mathrm{MAN}} \times S_{\mathrm{S}_{-} \mathrm{TD}}$ is dropped because researchers omit the specific thought disorder factor, the measurement equation reduces to $M A N=\alpha_{\text {MAN }}+\lambda_{\mathrm{P}, \mathrm{MAN}} \times P+\varepsilon_{\mathrm{MAN}}$. The observed mania score is now the sum of an intercept $\left(\alpha_{\mathrm{MAN}}\right)$, the "general" factor weighted with the factor loading of mania on $P$ $\left(\lambda_{\mathrm{P}, \mathrm{MAN}}\right)$ and measurement error $\varepsilon_{\mathrm{MAN}}$. In other words, the $P$ factor is now the true score variable pertaining to the mania indicator. It differs from mania only in terms of an intercept $\left(\alpha_{\text {MAN }}\right)$, a scaling constant (factor loading $\left.\lambda_{\mathrm{P}, \mathrm{MAN}}\right)$ and random measurement error $\left(\varepsilon_{\text {MAN }}\right)$.

If the entire $S$ factor collapses, that is, if all indicators have non-significant loadings on the $S$ factor or the $S$ factor shows a zero or negative variance estimate, $P$ becomes the common factor pertaining to mania and/or the other indicators of that $S$ factor. In our example, the general factor would be a thought disorder factor, that is, a latent variable underlying severity scores of mania, psychosis, and OCD.

Notice that the general factor can also have a more narrowly defined meaning. If only the $S$ factor loading of the mania indicator becomes non-significant, with factor loadings of OCD and psychosis on $P$ and $S$ remaining strong, the general factor depicts just mania. This makes clear why the meaning of $P$ changes depending on which $S$ factor collapses, is weakly defined, or omitted entirely. This underlines the fact that $P$ is not a general factor even if all indicators load on the factor. Instead, $P$ is defined by the indicators that only load on $P$ and not on the other factors. Consider a study in which a growth curve model is used to model change in depression severity across several measurement occasions. All depression severity scores load onto the intercept factor with the same loading of 1.0. The intercept factor is 
interpreted correctly as the severity of depression pertaining to the timepoint for which the slope factor loading is fixed to zero and not as a general factor of depression severity.

Similarly, $P$ is only interpretable as a general factor when all variables have both substantial $P$ and substantial $S$ factor loadings. We illustrate this issue in more detail in the empirical section below.

The example used to illustrate the measurement equation can be transferred to the results reported by Caspi et al. (2014). In that application, the specific thought disorder factor was dropped completely from the model due to a negative variance estimate. Therefore, their $P$ factor was no longer a general factor, but instead became a thought disorder factor. This is demonstrated by the fact that the mania and schizophrenia indicators (1) loaded particularly highly on $P$ (.97 and .82; the $P$ factor loading for mania was highest of all indicators) and (2) had no additional specific factor.

Caspi et al. (2014) provided further evidence that both latent variables were the same. Standardized factor loadings of indicators of the thought disorder factor were very similar in the model with correlated factors and in the bifactor model $(.73, .98$ and .83 versus $.73, .97$ and .82). Moreover, factor scores of the $P$-factor and the thought disorder factor showed a correlation of .997 , and correlations between the $P$-factor and external variables were similar in magnitude to correlations between the first-order thought disorder factor and external variables in the model with correlated factors. The average deviation of the absolute values of correlations across all tested associations was only .007 and there was no absolute difference larger than .017 .

The same arguments apply to other studies in which $S$ factors were omitted post hoc. For example, Romer et al. (2019) favored a bifactor model (i.e., no $S$ factor for thought disorder) and, similar to Caspi et al. (2014), found an extremely high correlation between $P$ factor scores and the factor scores of the thought disorder factor identified in the model with correlated factors (.98). This shows that Romer et al. (2019) tested the relationship between 
neocortical thickness and thought disorder and not necessarily the relationship between neocortical thickness and an overarching $P$.

Similarly, in one application reported by Tackett et al. (2013), the $S$ factor for major depressive disorder (MDD) and GAD was omitted from their bifactor model (see their Figure 3), making the general factor an MDD/GAD factor (MDD and GAD loaded .94 and .95 on the general factor, respectively). That factor might carry a similar (but not interchangeable) meaning as the general factor modeled in Brandes et al. (2019). In that study, items for withdrawn-depressed and major depressive disorder loaded exclusively on $P$, making the general factor a depressive mood factor.

In all previously cited studies, entire $S$ factors were omitted or $S$ factor loadings that were non-significant were fixed to zero. However, the same shift in interpretation occurs when one or more indicators have no substantial loadings on their $S$ factors but load highly on $P$, as in both the Lahey et al. (2012) and Watts et al. (2019) applications, where $S$ factors did not collapse completely, but several indicators had strong loadings only on $P$ and not on their $S$ factors.

Similarly, Martel et al. (2017) modeled general factors of psychopathology among children and their mothers. In each model, at least one item had a loading of zero or close to zero on one of the $S$ factors. Modeling $P$ in children, the $S$ factor loading of the autism spectrum indicator was fixed to zero, making the $P$ factor in children an autism factor. Modeling maternal $P$, the loading of the separation anxiety indicator on the $S$ factor for fear was very close to zero (-.006), giving the maternal $P$ factor the meaning of separation anxiety. Consequently, there was no consistency in the meaning of $P$ across groups (or studies).

Why Collapsing Factors Also Change the Meaning of $S$. Importantly, when a $S$ factor collapses, the meaning of the remaining $S$ factors also change. Given that $P$ is now defined based on indicator(s) with no $S$ factor (or zero $S$ factor loadings), the remaining $S$ factors reflect specific variance relative to the domain with no $S$ factor. For example, the $S$ 
factor for internalizing in Caspi et al. (2014) now indicates variance in the specific internalizing factor that is independent of the thought disorder factor rather than specific internalizing variance independent of a general $P$ factor. The same is true for the Romer et al. (2019) application. In Brandes et al. (2019), the specific internalization factor represents the part of internalization that cannot be predicted by depressed mood, and in Tackett et al. (2013), the specific internalization factor represents specific internalizing variance that was independent of the latent variable underlying MDD/GAD. Thus, the meaning of the $S$ factors also varies from study to study when using the $\mathrm{BF}_{\text {SYM }}$ approach.

In our experience, even though weak or collapsed $S$ factors (or individual variables without substantial $S$ factor loadings) are exceedingly common in empirical applications, researchers continue to interpret $P$ and $S$ in the same way as if all variables had substantial loadings on both $P$ and $S$ factors. That is, researchers continue interpreting $P$ as a general factor and $S$ factors as residuals with respect to the general factor. As we have shown above, such an interpretation is inapt and (however unintentionally) misleading when $S$ factors collapse or when $S$ factor loadings are zero or near zero. In the next section, we describe the distinction between interchangeable and structurally different domains and explain why this differentiation is crucial for selection of an appropriate bifactor model.

\section{Interchangeability as a Prerequisite for Meaningful General Factors}

Eid and Koch (2014) argued that, from the perspective of stochastic measurement theory, interchangeability of domains is an essential prerequisite for a properly interpretable general factor in BFSYM models and for avoiding anomalous results (see also Eid et al., 2017). Interchangeability requires a universe of domains from which a small subset of those domains is selected randomly. Domains represent random effects when interchanging any domain for another does not alter the meaning of the general factor, and when each combination of domains is equally well suited to represent the general factor. 
Interchangeability can be attained, for example, when researchers randomly sample situations from the life of a person and ask the person to evaluate his or her depressive mood on several items in each specific situation. When such ratings are modeled as a bifactor structure, the general factor represents expected depressive mood across situations and $S$ factors represent situation-specific deviations. The same applies when researchers randomly select friends from an individual's social network and ask each friend to assess how depressed the person is. The general factor represents that person's average depressive mood as perceived by their friends, and $S$ factors capture informant-specific deviations. Both examples represent a two-level measurement design with measurements nested within persons (Eid et al., 2017; Eid \& Koch, 2014; Geiser et al., 2012). Random sampling on both levels (i.e., persons and situations) ensures that the general factor is appropriately interpretable as an expected value across domains (Eid et al., 2017; Eid \& Koch, 2014). This sampling process implies that it does not matter which random sample of situations or random sample of friends we choose - an idea that is difficult (perhaps impossible) to transfer to symptoms or domains of psychopathology.

It seems more reasonable to assume that domains and symptoms of psychopathology are structurally different, and that each combination of symptoms provides a unique perspective on the individual's burden. Being structurally different also means that domains can have domain-specific vulnerabilities and can impair psycho-social functioning differently.

The fact that different domains of psychopathology are better regarded as structurally different is reflected empirically in inter-domain correlations that are often heterogeneous in magnitude. In Caspi et al. (2014), for example, latent correlations differed strongly between the three first-order factors with correlations of .33 between internalizing and externalizing factors, .85 between internalizing and thought disorder, and .58 between externalizing and thought disorder (see also Laceulle et al., 2015; Romer et al., 2018, 2019). For interchangeable domains, we expect these intercorrelations to be about equal. This is because 
sampling and measurement error are the only sources of dispersion around the sampling mean for exchangeable domains; there is no systematic structural difference between exchangeable domains that would cause some correlations to be substantially higher than others. In contrast, structurally different domains (e.g., internalizing, externalizing, thought disorder) differ systematically from one another, leading to heterogenous inter-domain correlations.

Geiser et al. (2015) showed that inadmissible results in BSYM models are more likely when models are fitted to structurally different domains. Therefore, the frequently encountered anomalous results in $P$-factor studies are probably best understood as a result of applying a modeling approach that requires interchangeable domains to structurally different domains. Preferably, we could select a modeling approach that (1) considers structural differences, (2) avoids anomalous results, and (3) gives the general factor an unambiguous meaning. We now present the $\mathrm{BF}_{S-1}$ approach as such an alternative.

\section{Bifactor-(S-1) Models and the Meaning of $P$ and $S$}

The $\mathrm{BF}_{S-1}$ approach was introduced as an alternative for estimating bifactor models designed specifically to account for structurally different domains (Eid et al., 2017). The major difference between the $\mathrm{BF}_{S Y M}$ model and the $\mathrm{BF}_{S-1}$ model is that the latter contains a subset of items (reference domain) which load exclusively on the general factor as a starting point (see Figure 1). The remaining items (pertaining to non-reference domains) load on the general factor and one $S$ factor. This structure ensures that both general and $S$ factors are unambiguous in their psychometric definition and interpretation.

In $\mathrm{BF}_{S-1}$ models, the factor labeled "general" does not represent an overarching dimension — even though all items load on it. The meaning of the general factor is instead defined $a$ priori by items that pertain to the reference domain, that is, items that load exclusively on the general factor. Take Model 3c, depicted in Figure 1, as an example. Items assessing thought disorder represent the reference domain. Thus, the general factor measures thought disorder and, most importantly, does so in the same manner as in the model with 
correlated first-order factors. The general factor and the corresponding first-order factor are equivalent - they depict the same latent variable (see Eid et al., 2017 for a more formal presentation; see also Burns et al., 2020a, Geiser et al., 2008, 2015; Heinrich et al., 2020).

The $\mathrm{BF}_{S-1}$ approach therefore makes specific use of the fact that items loading only on the general factor define its meaning. In the $\mathrm{BF}_{S-1}$ model, the general factor is defined a priori by selecting a theoretically meaningful reference domain. This contrasts with the BSYM model in which the data decide what the general factor represents. As a consequence, as we explained above, the meaning of $P$ in a B $\mathrm{BYM}_{\mathrm{SY}}$ model largely depends on which set of structurally different domains is included in a given study and which of these domains collapse.

When selecting a reference domain a priori, the psychometric definition and meaning of the general factor are clear: $P$ represents common true score variance reflected in the indicators of the reference domain (e.g., thought disorder symptoms). The meaning and interpretation of $P$ does not change when other domains are added to the model or when domains are removed from the model. As long as the reference domain remains the same, $P$ has the same meaning across studies that include different domains as $S$ factors. $S$ factors are clearly defined as residual factors with a mean of zero (Eid et al., 2017). These $S$ factors offer a cleaner interpretability than those of $\mathrm{BF}_{\mathrm{SYM}}$ models. They represent the part of a domain that cannot be explained by the reference facet, and not the part of a domain that cannot be explained by something that researchers do not know what it actually is, as is the case with $P$. Take Model 3c, depicted in Figure 1, again as an example. The thought disorder factor represents the reference domain; therefore, the $S$ factor for internalization represents that part of internalization that cannot be predicted linearly by thought disorder.

We explained earlier that correlations between $S$ factors in $\mathrm{BF}_{\mathrm{SYM}}$ models must be fixed to zero. In contrast, these correlations can be estimated and meaningfully interpreted in $\mathrm{BF}_{S-1}$ models. These associations are partial correlations, representing strengths of 
associations between first-order factors corrected, corrected for the influence of the reference domain (Eid et al., 2017; Geiser et al., 2008). In other words, they represent what two $S$ factors have in common once the effect of the reference domain has been partialled out. For example, the correlation between the $S$ factors for internalizing and externalizing reported by Caspi et al. (2014) show that internalizing and externalizing share variance above-and-beyond what both domains share with thought disorder.

\section{Illustrative Example}

We now illustrate the effect of collapsing factors on the meaning of the general factor in the $\mathrm{BF}_{S Y M}$ model, as well as fundamental properties of $\mathrm{BF}_{S-1}$ models based on an empirical example (for other applied examples see: Burns et al., 2020a; Demkowicz et al., 2019; Gäde et al., 2017; Heinrich et al., 2020; Junghänel et al., 2020). First, we show that the BF model produces anomalous results and that the general factor in the $\mathrm{BF}_{\mathrm{SYM}}$ model becomes the specific latent variable underlying the indicators of a collapsing factor. Second, we show it does not matter which $S$ factor is omitted to avoid anomalous results, but that selection of the omitted $S$ factor determines the meaning of the general factor. Last, we illustrate that, provided the reference domain of a $\mathrm{BF}_{S-1}$ model remains the same, the meaning of the general factor also remains the same, regardless of whether domains are added or removed.

We use data originating from an ongoing study in which we aim to construct a scale to allow individual symptoms of depressive disorders to be represented as latent variables. Each symptom is assessed with several items. Participants rated how often they experienced emotions and behaviors described in the items in the two weeks preceding assessment using a five point-rating scale ranging from never (0) to always (5). Categories 4 and 5 were collapsed because the category always was rarely endorsed for the indicators assessing low appetite. Items were taken from the Inventory of Depression and Anxiety Symptoms (IDAS, Watson et al., 2008, 2012; Watson \& O’Hara, 2017) and were complemented by additional items unless several items were available for assessment of a specific symptom. 
Participants were also encouraged to assess their sleep quality ("How restful was your sleep?"), rated on a 10-point scale with higher values indicating better sleep quality. In addition, we used an item from the stress module of the Patient Health Questionnaire (Löwe et al., 2004) asking participants how much they felt impaired due to stress at work/school in the four weeks preceding assessment. That item was rated on a 3-point scale ranging from not at all (0) to severely impaired (2). All self-report questionnaires were completed online. The sample consisted of 450 persons with an average age of 25.6 years $(S D=7.5$; range: 18 to 62). Most participants were female $(n=363,81 \%)$. Data collection was approved by the Ethics Committee of the Freie Universität Berlin.

All models presented below were estimated using the WLSMV estimation method implemented in Mplus version 8.3. Indicators of symptoms and the item assessing stress at work were treated as ordered categorical. Sleep quality was treated as a continuous variable. Output files including descriptive item statistics are available online (https://osf.io/sq4zd/).

\section{Symmetrical Bifactor Models}

For the first part of the illustrative example, we used the symptoms of sadness, low appetite, and concentration problems (each assessed with three items) and estimated a correlated factor model. We also tested a $\mathrm{BF}_{\mathrm{SYM}}$ model with uncorrelated $S$ factors.

Model Fit. Both models fitted the data well (see Table 1). As is typical in empirical applications (see above), fit of the BF $\mathrm{BSM}_{\mathrm{S}}$ model was superior to model fit for the model with correlated factors. This is to be expected because the BF $\mathrm{BSM}_{\mathrm{SY}}$ model is less restrictive (estimates more free parameters).

Correlations. Correlations between factors in the correlated factor model are summarized in Table 2. The correlation between the sadness and concentration factors was rather high (.74), whereas correlations between the sadness and the low appetite factors (.52) and between the low appetite and concentration factors (.40) were substantially lower. This pattern indicates that the three domains are not interchangeable but are structurally different 
as is typical in studies of $P$. For interchangeable domains, one would expect the correlations to be very similar.

Anomalous Results. In the BF $\mathrm{BYM}_{\mathrm{M}}$ model, the specific sadness factor collapsed. The standardized factor loadings of indicators assessing sadness were small and non-significant (standardized loadings: $\lambda_{\mathrm{SAD} 1}=.32, S E=.17, p=.062, \lambda_{\mathrm{SAD} 2}=.11, S E=.25, p=.661, \lambda_{\mathrm{SAD} 3}$ $=.21, S E=.27, p=.436)$. Factor variance of the specific sadness factor was also very small $(0.10, S E=0.11)$. Therefore, the $\mathrm{BF}_{S Y M}$ model became an empirical $\mathrm{BF}_{S-1}$ model with uncorrelated specific factors in which items assessing sadness defined the meaning of $P$. As is typical in empirical applications, we removed the collapsing specific factor for sadness. The resulting $\mathrm{BF}_{S-1}$ model with sadness as a reference domain fit the data well (see Table 1). We allowed the $S$ factors of the $\mathrm{BF}_{S-1}$ model to correlate because as we have described above, constraining these correlations would be unnecessary restrictive. In our example, the partial correlation between the specific concentration factor and the specific appetite factor was close to zero (.02) indicating that concentration problems and low appetite have nothing in common after sadness is partialled out. In order to illustrate that the factor sadness is in fact always the same in all models, we additionally modelled a one factor model using only the three indicators assessing sadness.

Results are summarized in Table 2 and support the idea that the general factor in our $\mathrm{BF}_{\mathrm{SYM}}$ model is a latent variable underlying the indicators of the collapsing factor. Standardized factor loadings of indicators assessing sadness are very similar in all models. In addition, the factor scores of the general factor in the original $\mathrm{BF}_{\mathrm{SYM}}$ model before any $S$ factor was dropped correlated almost perfectly with (1) factor scores of the reference factor in the $\mathrm{BF}_{S-1}$ model with sadness as reference domain and corelated $S$ factors (.99), (2) factor scores of the sadness factor in the model with correlated factors (.99), and (3) factor scores in the single-factor sadness model (.98). 
Correlation with Stress and Sleep Quality. Although we tried to estimate correlations of latent variables with stress and sleep quality, the BSYM model did not converge and the standard errors could not be computed. We therefore used factor scores from the previous models in which stress and sleep quality were not considered to examine the relationships between the general factor and the external variables. Correlations of the factor scores of general factor in the original $\mathrm{BF}_{\mathrm{SYM}}$ model before the specific sadness was factor was dropped with stress (.42) and sleep quality (-.54) were virtually identical to correlations of the general factor in the $\mathrm{BF}_{S-1}$ model with sadness as reference and correlated specific factors $(.41$ and -.54 , respectively). The same was true for the correlations of the sadness factor from the model with correlated factors (.41 and -.54 , respectively) and the single-factor model that included only sadness (.40 and -.52 , respectively).

Summary. High correlations between factor scores from the different models and the equivalence of correlations with stress and sleep quality support the assertion that when an $S$ factor collapses, the general factor in the $\mathrm{B}_{\mathrm{SYM}}$ model becomes the latent variable underlying the indicators with the non-significant or very small $S$ factor loadings. Researchers do not obtain the intended "overarching" factor, but instead model a $P$ factor that is empirically defined by a specific domain of psychopathology.

\section{Consequences of Omitting Different $S$ Factors}

In the first part of the illustrative example, we removed the specific sadness factor because indicators of that factor showed anomalous results. This decision was purely datadriven, as is typical in most applications of bifactor modeling. We now illustrate that we could have removed any other $S$ factor instead to avoid anomalous results. However, this results in different meanings of $P$ and remaining $S$ factors. To illustrate, we additionally estimated a $\mathrm{BF}_{S-1}$ model with low appetite as reference and a $\mathrm{BF}_{S-1}$ model with concentration problems as reference. We also examined how the interpretation of correlations changes when different $S$ factors are omitted. 
Model Fit. Regardless of which $S$ factor was removed, all models fit the data well (see Table 1).

Factor Loadings. All $\mathrm{BF}_{S-1}$ models provided reasonable parameter estimates. No anomalous results were encountered (see Table 2). Items that defined the reference domain in each of the $\mathrm{BF}_{S-1}$ models are highlighted in grey. Several points should be mentioned. First, the (un-) standardized factor loadings of indicators of the reference domain are equal in the $\mathrm{BF}_{S-1}$ model and in the model with correlated factors. Second, the variance of the latent variable representing the general factor in the $\mathrm{BF}_{S-1}$ model is the same as the variance of the corresponding factor in the correlated factors model. This set of findings indicates that the latent variables are the same. If the specific sadness factor is removed, the general factor reflects sadness. If the specific concentration problems factor is removed, the general factor reflects concentration problems. If the items that measure low appetite are used as a reference, the general factor reflects low appetite.

Correlations Between $S$ Factors. Correlations between the $S$ factors change depending on which domain is used as reference. Using low appetite as reference, the partial correlation between the specific sadness and concentration factors was large (.67). In contrast, the correlation between the specific concentration factor and the specific low appetite factor in a $\mathrm{BF}_{S-1}$ model with sadness as reference (.02), and the correlation between the specific appetite factor and the specific sadness factor in a BFS-1 model with concentration problems as reference (.37) were lower. These differences are to be expected, as the size of the correlations between the factors in the first-order correlated factor model also vary.

Correlation with Stress and Sleep Quality. Correlations of sleep quality and stress with latent variables are summarized in Table 3. A pattern is apparent: the correlations with the general factor always correspond to the correlations with the factor from the model with correlated factors if this factor is the one used as reference domain in the $\mathrm{BF}_{S-1}$ model. For example, the correlation between sadness and sleep quality in the model with correlated 
factors is moderately high (.43) and, most importantly, of the same size as the correlation between the general factor and sleep quality in the $\mathrm{BF}_{S-1}$ model with sadness as reference (.43). Correlations with the general factor, therefore, represent correlations with the reference domain. Correlations between the $S$ factors and external variables change depending on which reference domain is used. This is to be expected; these correlations are semi-partial correlations, as only the $S$ factors and not the external variables are controlled for the influence of the reference domain (this reasoning also applies to Caspi et al., 2014).

Summary. To summarize, we illustrated that no matter which $S$ factor is removed from the $\mathrm{BF}_{S-1}$ model, anomalous results are avoided. In addition, even though the meaning of the general factor changes depending on which domain serves as reference (i.e., which $S$ factor is omitted), the meaning of the general factor is clear in each version of the model: The general factor in this approach is the common factor that pertains to the reference domain. This underlines that the decision regarding which specific factor to remove should not be data-driven but should be based on a priori theoretical or substantive reasons. In addition, the general factor should not be interpreted as a general factor of psychopathology unless the items pertaining to the reference domain represent direct measures of general psychopathology.

\section{Consequences of Exchanging $S$ Factors in $\mathbf{B}_{S-1}$ Models}

Lastly, we show that as long as the reference domain remains the same in $\mathrm{BF}_{S-1}$ models, the meaning of the general factor is constant across applications that use different non-reference facets. For this illustration, we estimated an additional $\mathrm{BF}_{S-1}$ model that included domains of early awakening and decision problems instead of low appetite and problems in decision making.

Model Fit. The model fit of all estimated models is summarized in Table 1. All models fit the data reasonably well. 
Factor Loadings and Variances. Factor loadings of the three indicators assessing the reference factor (sadness) are very similar in both models (see Table 2). The same applies to variances of the factors (.81 and .82 , respectively). Correlations between factor scores of the general factors are high (.99), indicating that the latent variables are the same, even though the entire set of $S$ factors was exchanged.

Correlation with External Variables. Increased sadness is associated in the same way with decreased self-rated sleep quality $(-.55)$ and increased impairment due to stress at work (.43) in both $\mathrm{BF}_{S-1}$ models.

\section{Overall Summary of the Illustrative Example}

We showed that the general factor in a $\mathrm{BF}_{\text {SYM }}$ model with a collapsing $S$ factor is the same as (1) the corresponding first-order factor from the model with correlated factors, (2) the general factor of a $\mathrm{BF}_{S-1}$ model in which the collapsing factor is considered as reference, and (3) a single-factor model that only comprises the indicators of the vanishing $S$ factor. Thus, regardless of whether a reference domain is defined a priori (e.g., Burns et al., 2020a; Heinrich et al., 2020; Junghänel et al., 2020), S factors are removed based on empirical results (e.g., Caspi et al., 2014; Lahey et al., 2012; Tackett et al., 2013), or non-significant factor loadings lead to reduction of a $\mathrm{BF}_{S Y M}$ model to an empirical $\mathrm{BF}_{S-1}$ model (e.g., Gluschkoff et al., 2019; Martel et al., 2017), consequences for the change in interpretation of $P$ and $S$ are the same: The general factor is no longer interpretable as an "overarching" factor, but instead carries a meaning defined by a specific set of symptoms/domains, and the $S$ factors are contrasted against that factor. To avoid a sample-specific, data-driven result as to what the general factor depicts, researchers should define the general factor a priori using the $\mathrm{BF}_{S-1}$ approach. When doing so, meaning of the general factor remains the same, whether domains are added or removed. The $\mathrm{BF}_{S-1}$ model also allows for meaningful correlations between specific factors that represent partial correlations among domains after the reference domain has been partialled out. 
Discussion

Bifactor models are often used to investigate the structure of psychopathology. In this manuscript, we argued that $\mathrm{BF}_{\mathrm{SYM}}$ models are of limited usefulness for modeling $P$ because the interpretation of the general factor is typically ambiguous and lacks comparability across studies. We presented the $\mathrm{BF}_{S-1}$ approach as a reasonable alternative that avoids anomalous results and assigns each factor a well-defined a priori theory-based meaning and interpretation.

\section{Symmetrical Bifactor Models are of Limited Usefulness}

Consistent with work by others, we point out that the current practice of bifactor modeling is problematic (Bonifay et al., 2017; Burns et al., 2020a; Eid et al., 2017, 2018; Heinrich et al., 2020; Sellbom \& Tellegen, 2019; van Bork et al., 2017; Watts et al., 2019). From a statistical point of view, it is possible to add a general factor to any model that consists of several correlated first-order factors. Reduced restrictiveness of bifactor models almost always leads to improved model fit because minor misspecifications are masked (e.g., Geiser et al., 2015; Greene et al., 2019; Murray \& Johnson, 2013). However, an often underappreciated but important question is whether inclusion of a general factor leads to a more meaningful and interpretable model. If researchers use $\mathrm{BF}_{\mathrm{SYM}}$ models in conjunction with structurally different domains, we believe the answer to this question is clearly "no." Structurally different domains almost always lead to heterogeneous patterns of correlations in which certain domains are more highly correlated than others. This heterogeneous correlation structure causes inconsistent factor loadings and factor collapse in BFSYM models, leading to the general factor turning into a domain- or even symptom-specific factor.

Even in rare instances in which no anomalous results occur, we consider $\mathrm{BF}_{\mathrm{SYM}}$ inappropriate for modeling psychopathology. For example, anomalous results are less likely if correlations between structurally different domains are similar and artificially "mimic" interchangeability. However, whether domains are structurally different or not is not an 
empirical determination but a rather a conceptual one. Thus, empirical results provide at best an indication of how plausible the interchangeability assumption is. From this standpoint, application of a BF SYM to structurally different domains always leads to a general factor without clear meaning, even if a solution contains all admissible parameter estimates.

We do not know what this factor means beyond the fact that it accounts for correlations among different domains. This ambiguity cannot be resolved by relating $P$ to external variables. Using a BFSYM model, it remains unclear whether an association with the general factor is an association with general psychopathology or with something completely different. The same is true when the general factor is used as a predictor in a regression model. Does general psychopathology, a specific psychopathology, or something quite different explain variance in the dependent variable? None of these questions can be answered conclusively, which limits the value for testing substantive hypotheses about mechanisms underlying various mental disorders.

In addition, the meaning of $P$ in $\mathrm{BF}_{\mathrm{SYM}}$ models is unlikely to be invariant across studies that use different psychopathologies as domains and/or in which different symptoms drop their $S$ factor. Consider the previously cited $P$ studies. In Lahey et al. (2012), $P$ would be most appropriately described as a generalized anxiety/agoraphobia/panic factor, whereas in Caspi et al. (2014), Romer et al. (2019), and Laceulle et al. (2015), $P$ represented thought disorder. In Tackett et al. (2013) and Watts et al. (2019), $P$ was defined by both generalized anxiety and major depression. In Brandes et al. (2019), it was defined by two items assessing depressive mood. In Swales et al. (2020), $P$ represented a latent variable underlying attention/social/thought problems, and in Snyder et al. (2017), the general factor was defined by two indicators assessing hyperactivity and inattention. In Martel et al. (2017), it captured autism in children and separation anxiety in mothers. Thus, the meaning of $P$ varies from study to study. Naming all general factors as the "general factor of psychopathology" or " $P$ factor" misleadingly suggests a consistency where none exists. In this context, the common 
interpretation of $P$ as a "unifying" dimension across samples and forms of psychopathology will almost certainly lead the field down blind alleys and toward false conclusions about the nature of mental illness.

Indeed, correlations of $P$ with external variables are not comparable across studies, making a meaningful accumulation of scientific knowledge in systematic reviews and metaanalysis difficult if not impossible. For example, the correlation of neuroticism with $P$ found by Brandes et al. (2019) was almost twice as large (.81 vs .43) as the correlation reported by Caspi et al. (2014). However, in Brandes et al. (2019) the n-factor and the $P$-factor both represented depressed mood, whereas Caspi et al.'s (2014) $P$ factor depicted thought disorder. Although these findings may show that neuroticism is more strongly associated with depressed mood than with thought disorder, they do not yield a unifying $P$, much less show how strongly any such $P$ is associated with neuroticism.

The fact that almost every $P$-factor study uses a different set of psychological assessments poses additional problems. Simply finding a general factor in data sets that assess different domains of psychopathology with completely different assessments does not mean that the general factor of psychopathology has been identified or replicated. Different researchers have highlighted that psychological assessments are not interchangeable (e.g., Fried \& Nesse, 2015; Østergaard, 2018). Each scale contains specificity due to response format(s) and assessment procedures, and because they do not always assess the same symptoms. Even if there were a general factor of psychopathology and this factor could be represented in $\mathrm{BF}_{S Y M}$ models, it is unlikely that different measures would capture $P$ in the same manner. Worse, there is no way to test the measurement invariance if $P$ is always measured differently.

\section{Towards a Direct and Unambiguous Assessment of $P$}

Most studies of $P$ seek to identify a general factor that is extracted based on the entire set of symptoms. In contrast, the $\mathrm{BF}_{S-1}$ approach does not include a general overarching 
factor. Instead, it defines $P$ as the factor underlying indicators of the reference domain. This is a very different approach to studying a general psychopathology factor. However, trying to measure $P$ directly seems appealing, considering that researchers using BF $\mathrm{BYM}_{\mathrm{SM}}$ models usually do not find the intended general $P$ factor either, as we have demonstrated in this article. If researchers (1) have a theory about the meaning of $P$ and (2) use a measurement instrument that captures $P$ directly based on that meaning, problems such as lack of comparability and the interpretational ambiguities are resolved, the meaning in this case becomes transparent and replicable.

Two further aspects are worth mentioning. First, as has been emphasized elsewhere, bifactor- $(S-1)$ models are not the panacea for all issues related to modeling psychopathology (Burns et al., 2020b). $S-1$ models address the questions that BF $\mathrm{BYM}_{\mathrm{M}}$ models try to answer ("What underlies various domains of psychopathology") with the advantageous property that the search for meaning is not data-driven but that meaning is ensured by a careful selection of indicators for the reference facet (Burns et al., 2020b). Second, it can be argued that $S-1$ models are not a sensible approach for $P$-factor research. This reservation can be justified if researchers conceptualize $P$ in a way that is not compatible with the $S-1$ approach. In this case, however, one must bear in mind that the same caution should also apply to the many published $\mathrm{BF}_{\mathrm{SYM}}$ models that have become an empirical $S-1$ model due to collapsing $\mathrm{S}$ factors and non-significant $S$-factor loadings.

Of note, neuroticism (also referred to as negative affectivity and negative emotionality) appears to be a promising marker of $P$, given links to a wide range of psychopathologies including both internalizing and externalizing symptoms and their cooccurrence across the life span (Beauchaine \& Tackett, 2020; Brandes et al., 2019; Caspi et al., 2014; Olino et al., 2014; Tackett et al., 2013). If a researcher considers neuroticism to be at the core of the meaning of $P$, neuroticism, or a highly related construct (negative emotionality, negative affectivity) should be assessed directly. See also Caspi and Moffitt 
(2018) or Smith et al. (2020) for four possible ways to define $P$ consistent the $\mathrm{BF}_{S-1}$ model (i.e., diffuse unpleasant affective state, impulse control over emotions, deficits in certain intellectual functions, disordered thought). These definitions provide guidance in the selection of a suitable measurement instrument for direct assessment of $P$ and/or for the choice of the reference domain in the $\mathrm{BF}_{S-1}$ approach.

By assessing $P$ directly, one can ensure the general factor carries the same meaning in different studies. See Figure 2 for an illustration in which is negative affect (NA) is treated as reference. Suppose that Researchers A and B consider NA as the defining domain for $P$ and use NA as a marker for the reference domain. Researcher A is interested in the relationship between $P$, autism, and depression. Therefore, she represents both psychopathologies as $S$ factors. Researcher B proceeds in the same way, with the difference that he models $S$ factors for autism and ADHD. By using the same reference domain (NA) assessed with the same measurement instrument, both researchers give the general factor the same meaning, allowing them to accumulate knowledge and compare findings (see Figure 3).

\section{Recommendations for Selecting an Appropriate Bifactor Model}

Based on arguments outlined in this manuscript, we have developed a decision tree to help researchers choose a suitable bifactor modeling approach (Figure 3). These recommendations transfer ideas developed in multi-method measurement approaches (Eid, 2000; Eid \& Koch, 2014; Geiser et al., 2012) to bifactor structures used to model $P$, and to all contexts in which bifactor models are used. We are aware that not everyone shares our critical view of BFSYM models and considers the interchangeability of domains as a necessary prerequisite; we have tried to take that into account. Next, we will briefly describe each step of the decision tree.

Initially, one must decide whether bifactor structure is necessary to answer a specific research question. Using a bifactor model means giving up the clarity of a model with correlated factors, which should always be analyzed as a first step (Eid, 2020). If a bifactor 
model is required, one must determine whether domains can be considered interchangeable or not. If the answer is "no, the domains are structurally different," we recommend choosing a suitable reference domain and estimate a $\mathrm{BF}_{S-1}$ model. We argue that domains in clinical psychology are rarely if ever interchangeable and that correlated factor models or $\mathrm{BF}_{S-1}$ models are most useful for the vast majority of research questions. However, it is important to keep in mind that the choice of the reference domain should be made based on theory and substantive considerations rather than empirical model fit (Eid, 2000, 2020; Geiser et al., 2008; Heinrich et al., 2020).

If the answer to the question of whether domains are interchangeable is "yes, domains are interchangeable" or "I'm not sure," then estimating a BFSYM model is a tenable first step. Non-convergence may indicate that the model is inappropriate. If the model converges, estimated parameters should be examined critically. Are there insignificant factor loadings? Are there factor loadings with altered signs compared to the first-order model with correlated factors? Are there factors with a non-significant or negative variance estimate? Are there any other results that are difficult to explain or interpret? If the answer to any of these questions is "yes," the domains may be better viewed as structurally different. In that case, we would recommend using the $\mathrm{BF}_{S-1}$ model, and, if a meaningful reference domain is not available, a correlated factors model.

It is important to consider that additional parameter constraints and Bayesian

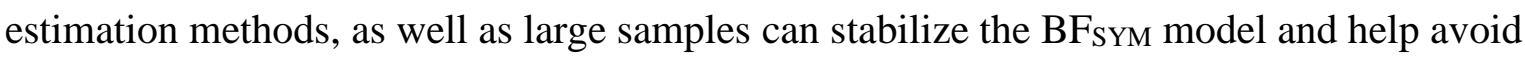
inadmissible parameter estimates. Such models converge, and at first glance appear appropriate, even when interpretational problems remain. In such situations, further evidence that the $\mathrm{BF}_{S Y M}$ bifactor model is a reasonable model should be provided. Here, the battery of tests proposed by Watts et al. (2019) offers a strategy to decide for or against a BFSYM model. We briefly summarize the proposed tests and describe what can be expected when a $\mathrm{BF}_{S-1}$ model is used (for comprehensive discussion see Watts et al., 2019). 
First, Watts et al. (2019) argue that the effect of the general factor on all indicators of all $S$ factors should be roughly the same. This consideration is important for BFSYM models, as researchers are forced to make the expected relation between the general factor and indicators transparent and test these expectations. This is rarely done. However, if a $\mathrm{BF}_{S-1}$ model is used, it is not necessary for all items to be equally linked to the general factor. Consider our empirical example, in which the correlation between sadness and concentration problems was stronger than the correlation between sadness and low appetite. Consequently, when sadness is used as reference, indicators of concentration problems will show higher loadings on the general factor than indicators of low appetite (see Table 2). That is, the size of factor loadings of the items of the non-reference domains can vary across the domains without indicating any problems in the $\mathrm{BF}_{S-1}$ approach.

Second, Watts et al. (2019) suggest that each $S$ factor should reflect an appropriate amount of reliable variance. This is consistent with other authors, who have argued that collapsing $S$ factors are problematic (Eid, 2000; Geiser et al., 2015). That test is particularly useful when the sample is large, where even small loadings are significant. Low variance in one of the $S$ factors indicates problems. For the $\mathrm{BF}_{S-1}$ model, however, the situation is different. In contrast to $\mathrm{BF}_{\mathrm{SYM}}$ models, low specificity does not provide evidence that the model should be rejected. Consider a factor model that includes the two highly correlated factors sadness and pessimism ( $r=.80,64 \%$ shared variance). Using sadness as a reference, the specific pessimism factor represents that part of pessimism that cannot be predicted by sadness, that is, what is unique after sadness is considered. Given shared features of sadness and pessimism, only a small amount of residual variance remains.

But even if no anomalous results occur and a model passes these tests, it is still unclear what the general factor in a $\mathrm{BF}_{\mathrm{SYM}}$ model measures because it is not defined by a specific set of indicators. To infer its meaning, researchers typically estimate correlations with external clinical variables and sometimes compare these correlations across studies. However, such 
comparisons are only meaningful when two bifactor models have the same structure. Proper consideration of comparability is particularly important if different measurement instruments are used. Because direct tests of measurement invariance are often not feasible, we recommend the following. First, researchers should investigate whether the models are specified in a similar manner (e.g., whether correlations between the $S$ factors are allowed). Second, they should check whether both models contain the same set of domains and whether these domains are measured using the same indicators. And third, they should verify whether the patterns of factor loadings are comparable. If two models differ in any of these aspects, it is unlikely the general factor has the same meaning and that correlations are comparable across studies.

\section{Limitations}

Some limitations of the present work should be considered. First, our arguments focused exclusively on bifactor models. Interchangeability is also a necessary prerequisite for meaningful interpretation of higher-order factors in a hierarchical factor analytic models (Eid et al., 2017). Second, we focus on interchangeability of domains as a critical feature to decide for or against specific variants of bifactor models. Of note, the meaning of bifactor models has also been scrutinized from perspectives other than those represented in the manuscript (e.g., van Bork et al., 2017). Third, we focus on modeling the general factor of psychopathology, but the same arguments apply to other applications aiming at modeling disorder-specific general factors, including general factors of depression, anxiety, ADHD, and all other mental disorders (e.g., Burns et al., 2020a; Heinrich et al., 2020; Junghänel et al., 2020). Fourth, we focus exclusively on the bifactor approach. Several other modeling approaches, such as formative measurement models and network models, have been proposed (see Caspi et al., 2018, for an overview). Fifth, all arguents presented above assume that indicators are homogeneous within their domain. Exemplarily, they assume that symptoms of mania, psychosis, and OCD are unidimensional indicators of the latent variable thought disorders. 
Whether this assumption is reasonable for thought disorder and other symptom domains is beyond the scope of this manuscript.

\section{Conclusion}

Widespread use of bifactor approaches to model the general factor of psychopathology must be viewed critically. Researchers who use $\mathrm{BF}_{\mathrm{SYM}}$ models often encounter high levels of ambiguity in the meaning of the modeled general factor. In most cases, these factors do not represent general psychopathology, but rather a specific domain of psychopathology. $\mathrm{BF}_{S-1}$ models are a useful alternative that circumvent many problems of $\mathrm{BF}_{S Y M}$ models. With this approach, the general factor has a clear meaning defined by the reference domain - though this factor should not be interpreted as the general factor of psychopathology unless $P$ is assessed directly. The $\mathrm{BF}_{S-1}$ approach allows for meaningful comparisons of $P$ across different informants, samples, time points, and studies. We hope that this manuscript will help researchers decide for or against the use of a bifactor structure, and to assess whether the use of a $\mathrm{BF}_{\mathrm{SYM}}$ model is truly the most fruitful approach to studying what different mental disorders have in common.

\section{References}

Beauchaine, T. P., \& Cicchetti, D. (2019). Emotion dysregulation and emerging psychopathology: A transdiagnostic, transdisciplinary perspective. Development and Psychopathology, 31(3), 799-804. https://doi.org/10.1017/S0954579419000671

Beauchaine, T. P., \& Tackett, J. L. (2020). Irritability as a transdiagnostic vulnerability trait: Currentissues and future directions. Behavior Therapy, 51(2), 350-364. https://doi.org/10.1016/j.beth.2019.10.009

Beauchaine, T. P., \& Zisner, A. (2017). Motivation, emotion regulation, and the latent structure of psychopathology: An integrative and convergent historical perspective. International Journal of Psychophysiology, 119, 108-118. https://doi.org/10.1016/j.ijpsycho.2016.12.014 
Bonifay, W., Lane, S. P., \& Reise, S. P. (2017). Three concerns with applying a bifactor model as a structure of psychopathology. Clinical Psychological Science, 5(1), 184186. https://doi.org/10.1177/2167702616657069

Brandes, C. M., Herzhoff, K., Smack, A. J., \& Tackett, J. L. (2019). The p factor and the $n$ factor: Associations between the general factors of psychopathology and neuroticism in children. Clinical Psychological Science, 2167702619859332. https://doi.org/10.1177/2167702619859332

Burns, G. L., Geiser, C., Servera, M., Becker, S. P., \& Beauchaine, T. P. (2020a). Application of the bifactor $\mathrm{S}-1$ model to multisource ratings of ADHD/ODD symptoms: An appropriate bifactor model for symptom ratings. Journal of Abnormal Child Psychology, 881-894. https://doi.org/10.1007/s10802-019-00608-4

Burns, G. L., Geiser, C., Servera, M., Becker, S. P., \& Beauchaine, T. P. (2020b). Promises and pitfalls of latent variable approaches to understanding psychopathology: Reply to Burke and Johnston, Eid, Junghänel and Colleagues, and Willoughby. Journal of Abnormal Child Psychology, 48(7), 917-922. https://doi.org/10.1007/s10802-02000656-1

Carragher, N., Teesson, M., Sunderland, M., Newton, N. C., Krueger, R. F., Conrod, P. J., Barrett, E. L., Champion, K. E., Nair, N. K., \& Slade, T. (2016). The structure of adolescent psychopathology: A symptom-level analysis. Psychological Medicine, 46(5), 981-994. https://doi.org/10.1017/S0033291715002470

Carver, C. S., Johnson, S. L., \& Timpano, K. R. (2017). Toward a functional view of the $p$ factor in psychopathology. Clinical Psychological Science, 5(5), 880-889. https://doi.org/10.1177/2167702617710037

Caspi, A., Houts, R. M., Belsky, D. W., Goldman-Mellor, S. J., Harrington, H., Israel, S., Meier, M. H., Ramrakha, S., Shalev, I., Poulton, R., \& Moffitt, T. E. (2014). The p Factor: One general psychopathology factor in the structure of psychiatric disorders? 
ON THE MEANING OF THE P-FACTOR

Clinical Psychological Science, 2(2), 119-137.

https://doi.org/10.1177/2167702613497473

Caspi, A., \& Moffitt, T. E. (2018). All for one and one for all: Mental disorders in one dimension. American Journal of Psychiatry, 175(9), 831-844. https://doi.org/10.1176/appi.ajp.2018.17121383

Demkowicz, O., Panayiotou, M., Ashworth, E., Humphrey, N., \& Deighton, J. (2019). The factor structure of the 4-Item Perceived Stress Scale in English adolescents. European Journal of Psychological Assessment, 1-5. https://doi.org/10.1027/1015$5759 / \mathrm{a} 000562$

Eid, M. (2000). A multitrait-multimethod model with minimal assumptions. Psychometrika, 65(2), 241-261. https://doi.org/10.1007/BF02294377

Eid, M. (2020). Multi-faceted constructs in abnormal psychology: Implications of the bifactor S - 1 model for individual clinical assessment. Journal of Abnormal Child Psychology. https://doi.org/10.1007/s10802-020-00624-9

Eid, M., Geiser, C., Koch, T., \& Heene, M. (2017). Anomalous results in G-factor models: Explanations and alternatives. Psychological Methods, 22(3), 541-562. https://doi.org/10.1037/met0000083

Eid, M., \& Koch, T. (2014). The meaning of higher-order factors in reflective-measurement models. Measurement: Interdisciplinary Research and Perspectives, 12(3), 96-101. https://doi.org/10.1080/15366367.2014.943591

Eid, M., Krumm, S., Koch, T., \& Schulze, J. (2018). Bifactor models for predicting criteria by general and specific factors: Problems of nonidentifiability and alternative solutions. Journal of Intelligence, 6(3), 42. https://doi.org/10.3390/jintelligence6030042

Fried, E. I., \& Nesse, R. M. (2015). Depression sum-scores don't add up: Why analyzing specific depression symptoms is essential. BMC Medicine, 13(1), 72. https://doi.org/10.1186/s12916-015-0325-4 
Gäde, J. C., Schermelleh-Engel, K., \& Klein, A. G. (2017). Disentangling the common variance of perfectionistic strivings and perfectionistic concerns: A bifactor model of perfectionism. Frontiers in Psychology, 8, 160. https://doi.org/10.3389/fpsyg.2017.00160

Geiser, C., Bishop, J., \& Lockhart, G. (2015). Collapsing factors in multitrait-multimethod models: Examining consequences of a mismatch between measurement design and model. Frontiers in Psychology, 6. https://doi.org/10.3389/fpsyg.2015.00946

Geiser, C., Eid, M., \& Nussbeck, F. W. (2008). On the meaning of the latent variables in the CT-C(M-1) model: A comment on Maydeu-Olivares and Coffman (2006). Psychological Methods, 13(1), 49-57. https://doi.org/10.1037/1082-989X.13.1.49

Geiser, C., Eid, M., West, S. G., Lischetzke, T., \& Nussbeck, F. W. (2012). A comparison of method effects in two confirmatory factor models for structurally different methods. Structural Equation Modeling: A Multidisciplinary Journal, 19(3), 409-436. https://doi.org/10.1080/10705511.2012.687658

Gluschkoff, K., Jokela, M., \& Rosenström, T. (2019). The general psychopathology factor: Structural stability and generalizability to within-individual changes. Frontiers in Psychiatry, 10. https://doi.org/10.3389/fpsyt.2019.00594

Gomez, R., Stavropoulos, V., Vance, A., \& Griffiths, M. D. (2019). Re-evaluation of the latent structure of common childhood disorders: Is there a general psychopathology factor (P-Factor)? International Journal of Mental Health and Addiction, 17(2), 258278. https://doi.org/10.1007/s11469-018-0017-3

Greene, A. L., Eaton, N. R., Li, K., Forbes, M. K., Krueger, R. F., Markon, K. E., Waldman, I. D., Cicero, D. C., Conway, C. C., Docherty, A. R., Fried, E. I., Ivanova, M. Y., Jonas, K. G., Latzman, R. D., Patrick, C. J., Reininghaus, U., Tackett, J. L., Wright, A. G. C., \& Kotov, R. (2019). Are fit indices used to test psychopathology structure 
ON THE MEANING OF THE P-FACTOR

biased? A simulation study. Journal of Abnormal Psychology, 128(7), 740-764. https://doi.org/10.1037/abn0000434

Haltigan, J. D., Aitken, M., Skilling, T., Henderson, J., Hawke, L., Battaglia, M., Strauss, J., Szatmari, P., \& Andrade, B. F. (2018). “P” and “DP:” Examining symptom-level bifactor models of psychopathology and dysregulation in clinically referred children and adolescents. Journal of the American Academy of Child \& Adolescent Psychiatry, 57(6), 384-396. https://doi.org/10.1016/j.jaac.2018.03.010

Heinrich, M., Zagorscak, P., Eid, M., \& Knaevelsrud, C. (2020). Giving G a meaning: An application of the bifactor-(S-1) approach to realize a more symptom-oriented modeling of the Beck Depression Inventory-II. Assessment, 27(7). https://doi.org/10.1177/1073191118803738

Holzinger, K. J., \& Swineford, F. (1937). The Bi-factor method. Psychometrika, 2(1), 41-54. https://doi.org/10.1007/BF02287965

Junghänel, M., Rodenacker, K., Dose, C., \& Döpfner, M. (2020). Applying the bifactor S-1 model to ratings of ADHD/ODD symptoms: A commentary on Burns et al. (2019) and a re-analysis. Journal of Abnormal Child Psychology. https://doi.org/10.1007/s10802020-00637-4

Laceulle, O. M., Vollebergh, W. A. M., \& Ormel, J. (2015). The structure of psychopathology in adolescence: Replication of a general psychopathology factor in the TRAILS study. Clinical Psychological Science, 3(6), 850-860.

https://doi.org/10.1177/2167702614560750

Lahey, B. B., Applegate, B., Hakes, J. K., Zald, D. H., Hariri, A. R., \& Rathouz, P. J. (2012). Is there a general factor of prevalent psychopathology during adulthood? Journal of Abnormal Psychology, 121(4), 971-977. https://doi.org/10.1037/a0028355

Lahey, B. B., Zald, D. H., Perkins, S. F., Villalta-Gil, V., Werts, K. B., Van Hulle, C. A., Rathouz, P. J., Applegate, B., Class, Q. A., Poore, H. E., Watts, A. L., \& Waldman, I. 
D. (2018). Measuring the hierarchical general factor model of psychopathology in young adults. International Journal of Methods in Psychiatric Research, 27(1), e1593. https://doi.org/10.1002/mpr.1593

Löwe, B., Kroenke, K., Herzog, W., \& Gräfe, K. (2004). Measuring depression outcome with a brief self-report instrument: Sensitivity to change of the Patient Health Questionnaire (PHQ-9). Journal of Affective Disorders, 81(1), 61-66. https://doi.org/10.1016/S01650327(03)00198-8

Markon, K. E. (2019). Bifactor and hierarchical models: Specification, inference, and interpretation. Annual Review of Clinical Psychology, 15(1), 51-69. https://doi.org/10.1146/annurev-clinpsy-050718-095522

Martel, M. M., Pan, P. M., Hoffmann, M. S., Gadelha, A., do Rosário, M. C., Mari, J. J., Manfro, G. G., Miguel, E. C., Paus, T., Bressan, R. A., Rohde, L. A., \& Salum, G. A. (2017). A general psychopathology factor (P factor) in children: Structural model analysis and external validation through familial risk and child global executive function. Journal of Abnormal Psychology, 126(1), 137-148. https://doi.org/10.1037/abn0000205

Murray, A. L., \& Johnson, W. (2013). The limitations of model fit in comparing the bi-factor versus higher-order models of human cognitive ability structure. Intelligence, 41(5), 407-422. https://doi.org/10.1016/j.intell.2013.06.004

Olino, T. M., Dougherty, L. R., Bufferd, S. J., Carlson, G. A., \& Klein, D. N. (2014). Testing models of psychopathology in preschool-aged children using a structured interviewbased assessment. Journal of Abnormal Child Psychology, 42(7), 1201-1211. https://doi.org/10.1007/s10802-014-9865-X

Østergaard, S. D. (2018). Do not blame the SSRIs: Blame the Hamilton Depression Rating Scale. Acta Neuropsychiatrica, 30(5), 241-243. https://doi.org/10.1017/neu.2017.6 
Reise, S. P. (2012). TherRediscovery of bifactor measurement models. Multivariate Behavioral Research, 47(5), 667-696. https://doi.org/10.1080/00273171.2012.715555 Romer, A. L., Elliott, M. L., Knodt, A. R., Sison, M. L., Ireland, D., Houts, R., Ramrakha, S., Poulton, R., Keenan, R., Melzer, T. R., Moffitt, T. E., Caspi, A., \& Hariri, A. R. (2019). A pervasively thinner neocortex is a transdiagnostic feature of general psychopathology. BioRxiv, 788232. https://doi.org/10.1101/788232

Romer, A. L., Knodt, A. R., Houts, R., Brigidi, B. D., Moffitt, T. E., Caspi, A., \& Hariri, A. R. (2018). Structural alterations within cerebellar circuitry are associated with general liability for common mental disorders. Molecular Psychiatry, 23(4), 1084-1090. https://doi.org/10.1038/mp.2017.57

Sellbom, M., \& Tellegen, A. (2019). Factor analysis in psychological assessment research: Common pitfalls and recommendations. Psychological Assessment, 31(12), 14281441. https://doi.org/10.1037/pas0000623

Smith, G. T., Atkinson, E. A., Davis, H. A., Riley, E. N., \& Oltmanns, J. R. (2020). The General Factor of Psychopathology. 16, 75-98. https://doi.org/10.1146/annurevclinpsy-071119-115848

Snyder, H. R., Young, J. F., \& Hankin, B. L. (2017). Strong homotypic continuity in common psychopathology-, onternalizing-, and externalizing-specific factors over time in adolescents. Clinical Psychological Science, 5(1), 98-110. https://doi.org/10.1177/2167702616651076

Swales, D. A., Snyder, H. R., Hankin, B. L., Sandman, C. A., Glynn, L. M., \& Davis, E. P. (2020). Maternal depressive symptoms predict general liability in child psychopathology. Journal of Clinical Child \& Adolescent Psychology, 1-12. https://doi.org/10.1080/15374416.2020.1723598

Tackett, J. L., Lahey, B. B., van Hulle, C., Waldman, I., Krueger, R. F., \& Rathouz, P. J. (2013). Common genetic influences on negative emotionality and a general 
psychopathology factor in childhood and adolescence. Journal of Abnormal Psychology, 122(4), 1142-1153. https://doi.org/10.1037/a0034151

van Bork, R., Epskamp, S., Rhemtulla, M., Borsboom, D., \& van der Maas, H. L. J. (2017). What is the p-factor of psychopathology? Some risks of general factor modeling. Theory \& Psychology, 27(6), 759-773. https://doi.org/10.1177/0959354317737185

Watson, D., \& O'Hara, M. W. (2017). Understanding the emotional disorders: A symptomlevel approach based on the IDAS-II. Oxford University Press.

Watson, D., O’Hara, M. W., Chmielewski, M., McDade-Montez, E. A., Koffel, E., Naragon, K., \& Stuart, S. (2008). Further validation of the IDAS: Evidence of convergent, discriminant, criterion, and incremental validity. Psychological Assessment, 20(3), 248-259. https://doi.org/10.1037/a0012570

Watson, D., O’Hara, M. W., Naragon-Gainey, K., Koffel, E., Chmielewski, M., Kotov, R., Stasik, S. M., \& Ruggero, C. J. (2012). Development and validation of new anxiety and bipolar symptom scales for an expanded version of the IDAS (the IDAS-II). Assessment, 19(4), 399-420. https://doi.org/10.1177/1073191112449857

Watts, A. L., Poore, H. E., \& Waldman, I. D. (2019). Riskier tests of the validity of the bifactor model of psychopathology. Clinical Psychological Science, 7(6), 1285-1303. https://doi.org/10.1177/2167702619855035 


\section{Footnotes}

${ }^{1}$ It should be noted that the size of the partial correlations can be determined by hand, by simply using the factor inter-correlations of the model with correlated factors and the formula for partial correlations. For example, in the correlated factor model, the correlation between sadness and low appetite is .52 , between sadness and concentration problems is .74 , and between low appetite and concentration problems .40. Using the formula for partial correlations and computing the partial correlation between sadness and concentration problems while treating low appetite as reference, we obtain

$$
r_{(S A D, C O N) \cdot L A P}=\frac{\left(r_{S A D, C O N}-r_{L A P, S A D} \times r_{L A P, C O N}\right)}{\sqrt{\left[\left(1-r_{L A P, S A D}^{2}\right) \times\left(1-r_{L A P, C O N}^{2}\right)\right]}}=\frac{.74-.52 \times .40}{\sqrt{\left(1-.52^{2}\right) \times\left(1-.40^{2}\right)}}=.68
$$

which corresponds well to the correlation between specific factors observed in the $\mathrm{BF}_{S-1}$ model (.67). This again highlights that the non-reference domains are in contrast with the reference factor and that the general factor carries the meaning of the reference domain. 
Table 1

Model Fit

\begin{tabular}{|c|c|c|c|c|c|c|c|}
\hline Model & $\chi^{2}$ & df & $p$ & RMSEA & {$[90 \% \mathrm{CI}]$} & CFI & SRMR \\
\hline CFA SAD, LAP, CON & 45.28 & 24 & .005 & .044 & {$[.024, .064]$} & .998 & .023 \\
\hline BF $_{S Y M}, S A D, L A P, C O N$ & 23.42 & 18 & .175 & .026 & {$[.000, .052]$} & .999 & .015 \\
\hline $\mathrm{BF}_{S-1}$, SAD, $S$-LAP, S-CON & 27.17 & 20 & .131 & .028 & {$[.000, .053]$} & .999 & .017 \\
\hline $\mathrm{BF}_{S-1}$, LAP, S-SAD, S-CON & 31.30 & 20 & .051 & .035 & {$[.000, .058]$} & .999 & .018 \\
\hline $\mathrm{BF}_{S-1}$, CON, S-SAD, S-LAP & 40.23 & 20 & .005 & .047 & {$[.026, .069]$} & .998 & .020 \\
\hline $\mathrm{BF}_{S-1}$, SAD, $S$-EAR, $S$-DES & 24.86 & 20 & .207 & .023 & {$[.000, .049]$} & 1.000 & .014 \\
\hline CFA SAD, LAP, CON + Stress + Sleep Quality & 59.76 & 36 & .008 & .038 & {$[.020, .055]$} & .998 & .021 \\
\hline 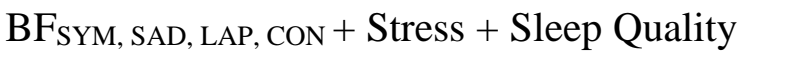 & dnc. & & & & & & \\
\hline $\mathrm{BF}_{S-1}$, SAD, $S$-LAP, $S$-CON + Stress + Sleep Quality & 41.10 & 32 & .130 & .025 & {$[.000, .045]$} & .999 & .017 \\
\hline $\mathrm{BF}_{S-1}$, LAP, $S$-SAD, $S$-CON + Stress + Sleep Quality & 45.35 & 32 & .059 & .030 & {$[.000, .049]$} & .999 & .017 \\
\hline $\mathrm{BF}_{S-1}, \mathrm{CON}, S$-SAD, $S$-LAP + Stress + Sleep Quality & 54.99 & 32 & .007 & .040 & {$[.021, .057]$} & .998 & .019 \\
\hline $\mathrm{BF}_{S-1}$, SAD, $S$-EAR, $S$-DES + Stress + Sleep Quality & 47.81 & 32 & .036 & .033 & {$[.009, .052]$} & .999 & .017 \\
\hline
\end{tabular}

Note $. \mathrm{df}=$ degrees of freedom. RMSEA $=$ Root Mean Square Error of Approximation. $\mathrm{CI}=$ Confidence Interval. $\mathrm{CFI}=$ Comparative Fit Index. SRMR = Standardized Root Mean Residual. dnc. = did not converge. SAD = sadness. LAP = Low Appetite. DES $=$ Problems in Decision Making. EAR = early awakening. $\mathrm{CON}=$ concentration problems. 
Table 2

Factor Loadings of the Correlated Factor Model as well as the Bifactor-(S-1) Model with Different Reference Domains.

\begin{tabular}{|c|c|c|c|c|c|c|c|c|c|c|c|c|c|}
\hline \multirow[t]{2}{*}{ Factor } & \multirow{2}{*}{$\begin{array}{l}\text { SFM } \\
\lambda\end{array}$} & \multirow{2}{*}{$\begin{array}{l}\text { CFA } \\
\lambda\end{array}$} & \multirow{2}{*}{$\begin{array}{l}\mathrm{BF}_{\mathrm{SYM}} \\
\lambda_{\mathrm{G}}\end{array}$} & \multirow[b]{2}{*}{$\lambda_{\mathrm{s}}$} & \multicolumn{2}{|c|}{$\begin{array}{l}\mathrm{BF}_{S-1} \\
\mathrm{SAD}_{S-\mathrm{LAP}, S-\mathrm{CON}}\end{array}$} & \multicolumn{2}{|c|}{$\begin{array}{l}\mathrm{BF}_{S-1} \\
\mathrm{LAP}_{S-\mathrm{SAD}, S-\mathrm{CON}}\end{array}$} & \multicolumn{2}{|c|}{$\begin{array}{l}\mathrm{BF}_{S-1} \\
\mathrm{CON}_{S \text {-LAP, } S \text {-SAD }}\end{array}$} & \multicolumn{3}{|c|}{$\begin{array}{l}\mathrm{BF}_{S-1} \\
\mathrm{SAD}_{S \text {-EAR, } S \text {-DES }}\end{array}$} \\
\hline & & & & & $\lambda_{\mathrm{G}}$ & $\lambda_{\mathrm{s}}$ & $\lambda_{\mathrm{G}}$ & $\lambda_{\mathrm{S}}$ & $\lambda_{\mathrm{G}}$ & $\lambda_{\mathrm{S}}$ & & $\lambda_{\mathrm{G}}$ & $\lambda_{\mathrm{S}}$ \\
\hline SAD & $1.00(.91)$ & $1.00(.90)$ & $1.00(.86)$ & $1.00\left(.32^{\mathrm{NS}}\right)$ & $1.00(.90)$ & & $0.48(.45)$ & $1.00(.79)$ & $0.78(.66)$ & $1.00(.62)$ & SAD & $1.00(.90)$ & \\
\hline SAD & $1.05(.95)$ & $1.07(.96)$ & $1.11(.96)$ & $0.34^{\mathrm{NS}}\left(.11^{\mathrm{NS}}\right)$ & $1.07(.96)$ & & $0.58(.54)$ & $1.01(.79)$ & $0.84(.71)$ & $1.03(.64)$ & SAD & $1.05(.95)$ & \\
\hline SAD & $1.02(.93)$ & $1.03(.92)$ & $1.04(.89)$ & $0.66^{\mathrm{NS}}\left(.21^{\mathrm{NS}}\right)$ & $1.03(.92)$ & & $0.51(.47)$ & $1.01(.80)$ & $0.80(.68)$ & $1.01(.63)$ & SAD & $1.02(.92)$ & \\
\hline LAP & & $1.00(.92)$ & $0.61(.53)$ & $1.00(.75)$ & $0.57(.52)$ & $1.00(.75)$ & $1.00(.92)$ & & $0.49(.41)$ & $1.00(.82)$ & EAR & $0.34(.31)$ & $1.00(.87)$ \\
\hline LAP & & $0.98(.91)$ & $0.55(.47)$ & $1.04(.78)$ & $0.52(.46)$ & $1.04(.78)$ & $0.98(.91)$ & & $0.41(.35)$ & $1.03(.84)$ & EAR & $0.36(.32)$ & $1.04(.90)$ \\
\hline LAP & & $0.98(.91)$ & $0.54(.46)$ & $1.07(.79)$ & $0.50(.45)$ & $1.06(.80)$ & $0.98(.91)$ & & $0.40(.34)$ & $1.05(.86)$ & EAR & $0.40(.36)$ & $0.92(.80)$ \\
\hline $\mathrm{CON}$ & & $1.00(.85)$ & $0.70(.61)$ & $1.00(.61)$ & $0.66(.59)$ & $1.00(.62)$ & $0.31(.29)$ & $1.00(.81)$ & $1.00(.85)$ & & DES & $0.77(.70)$ & $1.00(.59)$ \\
\hline $\mathrm{CON}$ & & $1.13(.96)$ & $0.83(.71)$ & $1.07(.65)$ & $0.78(.70)$ & $1.08(.67)$ & $0.41(.38)$ & $1.08(.88)$ & $1.13(.96)$ & & DES & $0.70(.63)$ & $1.00(.59)$ \\
\hline $\mathrm{CON}$ & & $1.02(.86)$ & $0.80(.69)$ & $0.80(.49)$ & $0.75(.67)$ & $0.81(.51)$ & $0.43(.40)$ & $0.93(.75)$ & $1.02(.86)$ & & DES & $0.79(.72)$ & $1.07(.63)$ \\
\hline \multicolumn{14}{|c|}{ Variances (Standard Deviations) } \\
\hline G & & & $0.74(1)$ & & & & & & & & & & \\
\hline SAD & $0.82(1)$ & $0.81(1)$ & $0.10^{\mathrm{NS}}(1)$ & & $0.81(1)$ & & $0.62(1)$ & & $0.38(1)$ & & & $0.82(1.00)$ & \\
\hline LAP & & $0.85(1)$ & $0.56(1)$ & & $0.57(1)$ & & $0.85(1)$ & & $0.67(1)$ & & & $0.75(1.00)$ & \\
\hline $\mathrm{CON}$ & & $0.72(1)$ & $0.37(1)$ & & $0.39(1)$ & & $0.66(1)$ & & $0.72(1)$ & & & $0.35(1.00)$ & \\
\hline \multicolumn{14}{|c|}{ Covariances (Correlations) } \\
\hline SAD, LAP & & $0.43(.52)$ & F0 & & F0 & & F0 & & $0.18(.37)$ & & EAR, DES & $0.04^{\mathrm{NS}}(.07$ & \\
\hline $\mathrm{SAD}, \mathrm{CON}$ & & $0.56(.74)$ & F0 & & F0 & & $0.43(.67)$ & & F0 & & & & \\
\hline LAP, CON & & $0.31(.40)$ & F0 & & $0.01^{\mathrm{NS}}(.02$ & $\left.{ }^{\mathrm{NS}}\right)$ & F0 & & F0 & & & & \\
\hline
\end{tabular}

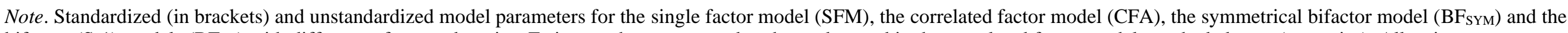

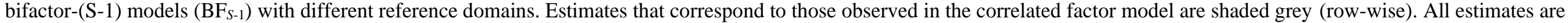

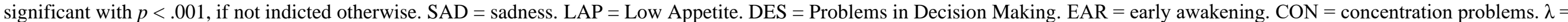

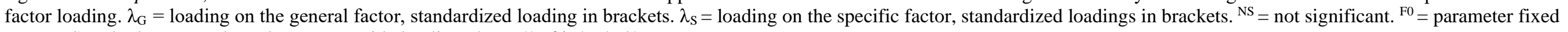
to zero. Standard errors and $p$ values are provided online: https://osf.io/sq4zd/. 
Table 3

Covariances and Correlations of Stress and Sleep Quality with the Latent Variables (SAD, LAP, CON).

\begin{tabular}{|c|c|c|c|c|c|c|}
\hline \multirow[t]{2}{*}{ Model } & \multicolumn{3}{|c|}{ Stress at Work } & \multicolumn{3}{|l|}{ Sleep Quality } \\
\hline & SAD & LAP & $\mathrm{CON}$ & SAD & LAP & $\mathrm{CON}$ \\
\hline 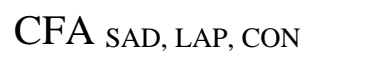 & $0.38(.43)$ & $0.19(0.20)$ & $0.37(.44)$ & $-1.14(-.55)$ & $-0.90(-.42)$ & $-0.94(-.48)$ \\
\hline $\mathrm{BF}_{S-1}$, SAD, $S$-LAP, $S$-CON & $0.38(.43)$ & $-0.02(-.03)$ & $0.12(.19)$ & $-1.14(-.55)$ & $-0.28(-.16)$ & $-0.17(-.12)$ \\
\hline $\mathrm{BF}_{S-1}$, LAP, $S$-SAD, $S$-CON & $0.29(.38)$ & $0.19(.20)$ & $0.32(.39)$ & $-0.70(-.39)$ & $-0.90(-.42)$ & $-0.64(-.34)$ \\
\hline $\mathrm{BF}_{S-1}, \mathrm{CON}, S$-SAD, $S$-LAP & $0.09(.15)$ & $0.02(0.03)$ & $0.37(.44)$ & $-0.42(-.29)$ & $-0.47(-.25)$ & $-0.94(-.48)$ \\
\hline
\end{tabular}

Note. Estimates that correspond to each other across the different models are shaded grey (please read column-wise). $\mathrm{SAD}=$ sadness. $\mathrm{CON}=$ concentration problems. $\mathrm{LAP}=$ low appetite. Standard errors and $p$ values are provided online: https://osf.io/sq4zd/. 


\section{Figure Captions}

Figure 1. Different models for three items assessing internalizing (INT1-INT3), externalizing (EXT1-EXT3) and though disorders (TD1-TD3). Model 1: Correlated factor model: Each Item loads on one domain specific first-order factor and all factors correlate with each other. Model 2: A symmetrical bifactor model: Each item loads on one specific factor, as well as on the general factor. The dotted line indicates correlations, which are allowed in many empirical applications, but are inadmissible and should be avoided. Model 3a to Model 3c: Bifactor- $(S-1)$ model: Each item loads on the general factor. Items, which do not belong to the reference domain, also load on one specific factor.

Figure 2. Two different bifactor- $(S-1)$ models with indicators that assess negative affectivity (NA) as markers for the reference domain. Thus, the general factors have the same meaning in both models. While Researcher A is interested in how much variance shared between autism spectrum disorder (ASD) and depression (DEP) can be explained by NA, Researcher B is interested in how much variance shared between attentiondeficit/hyperactivity disorder (ADHD) and ASD can be explained by NA. The partial correlations between the specific factor indicate, what both factors have in common once the effect of NA has been partialled out. As both researchers use the same reference, they can compare their results and accumulate knowledge.

Figure 3. Decision tree to decide between different variants of bifactor structures. 


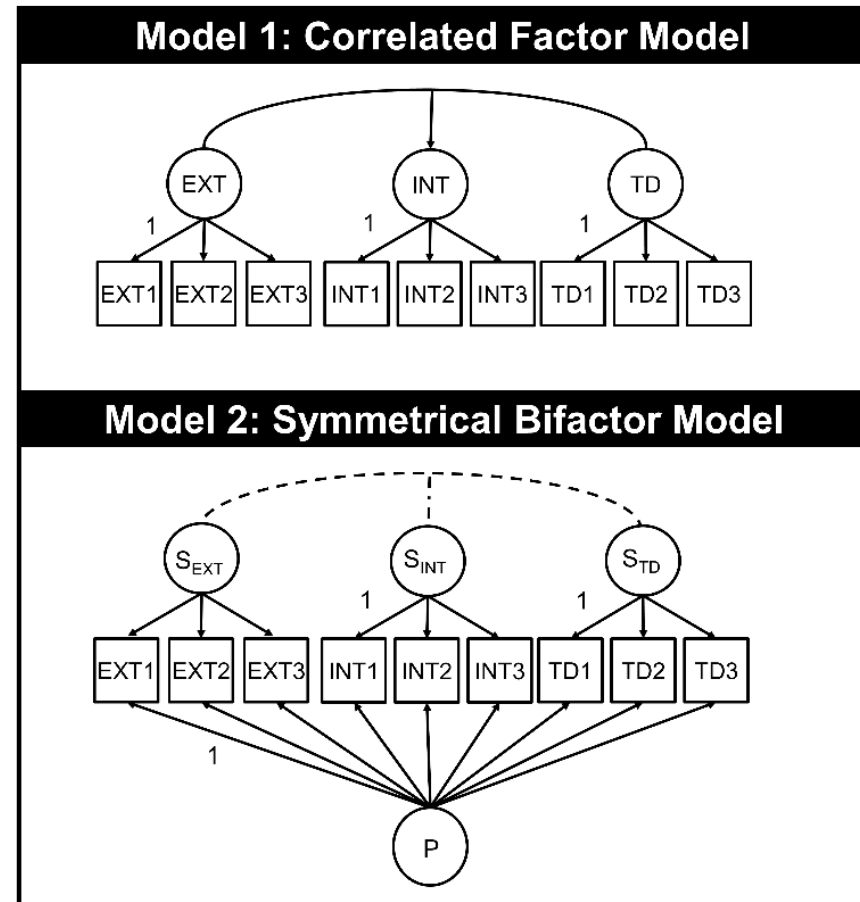

\section{Model 3a: Bifactor-(S - 1), EXT as reference}

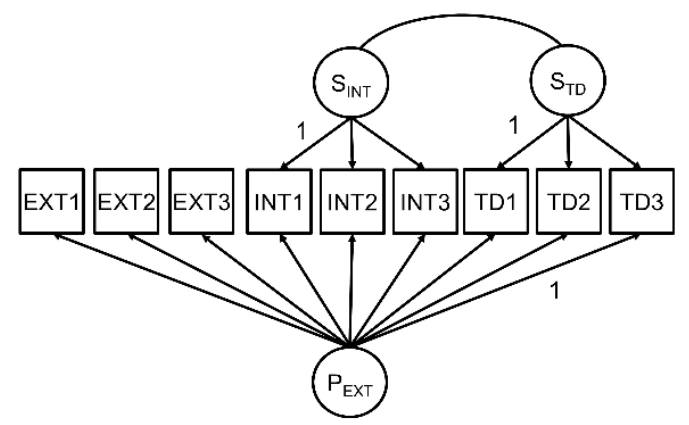

Model 3b: Bifactor-(S - 1), INT as reference

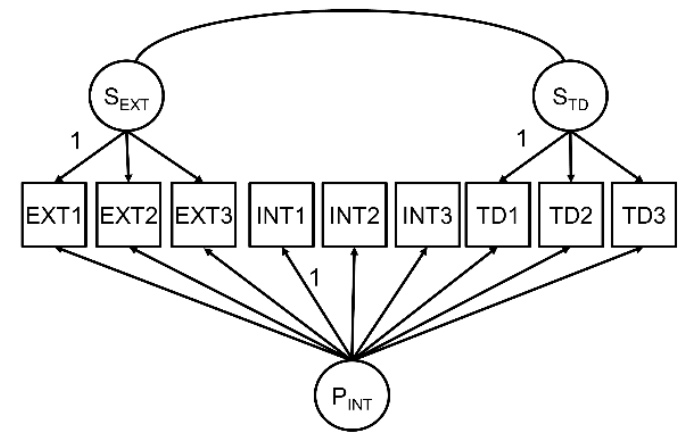

Model 3c: Bifactor-(S - 1), TD as reference

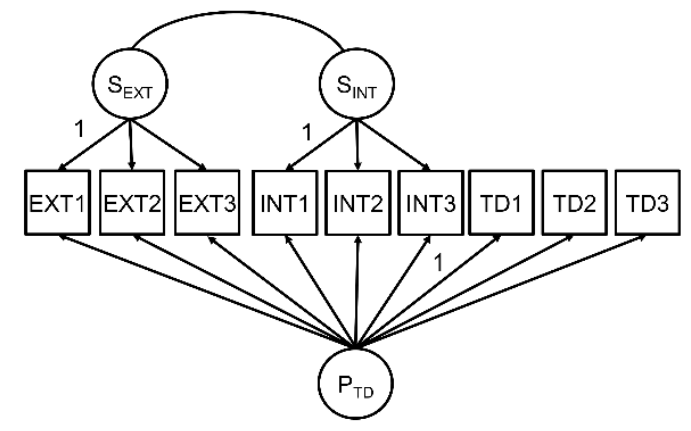




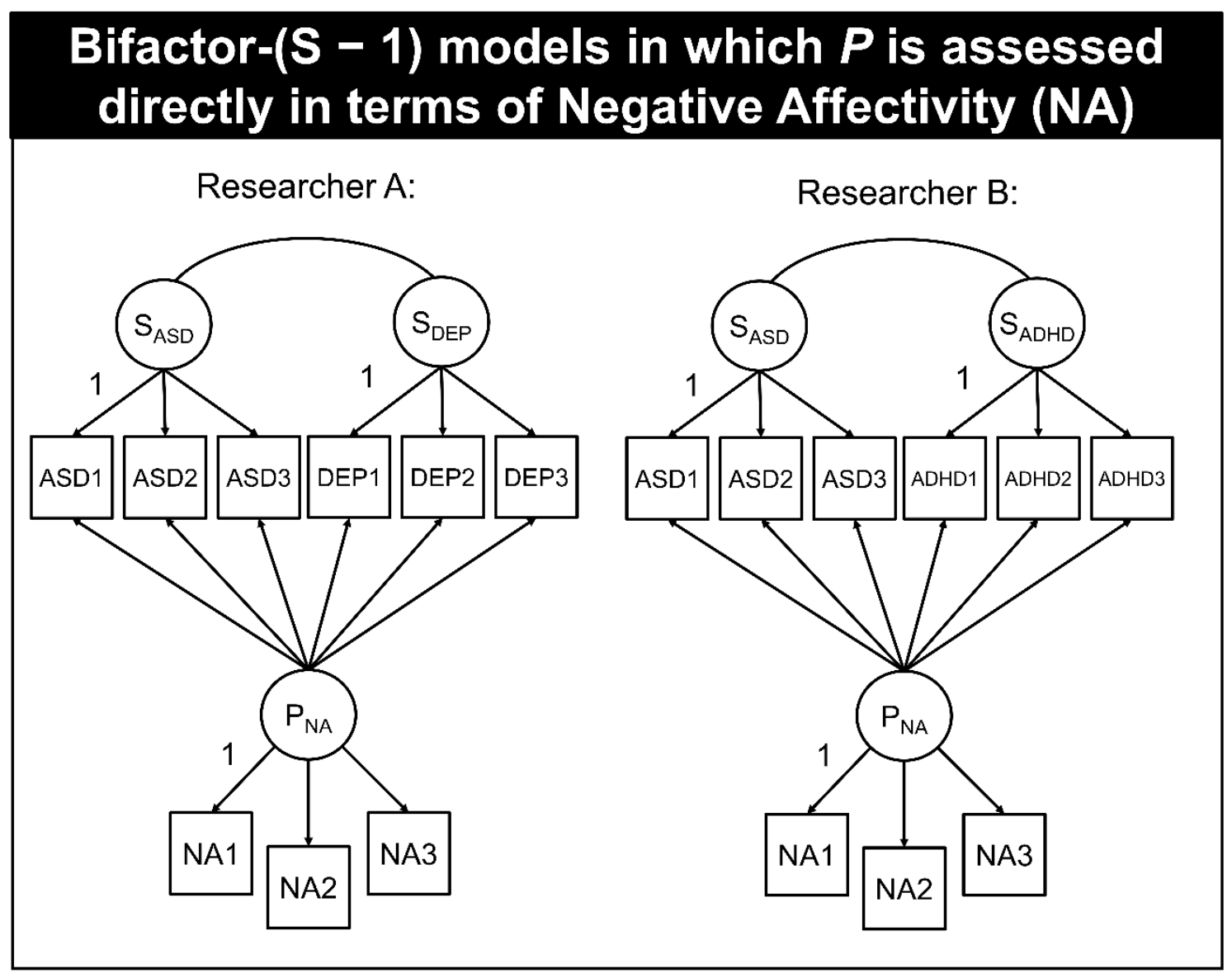




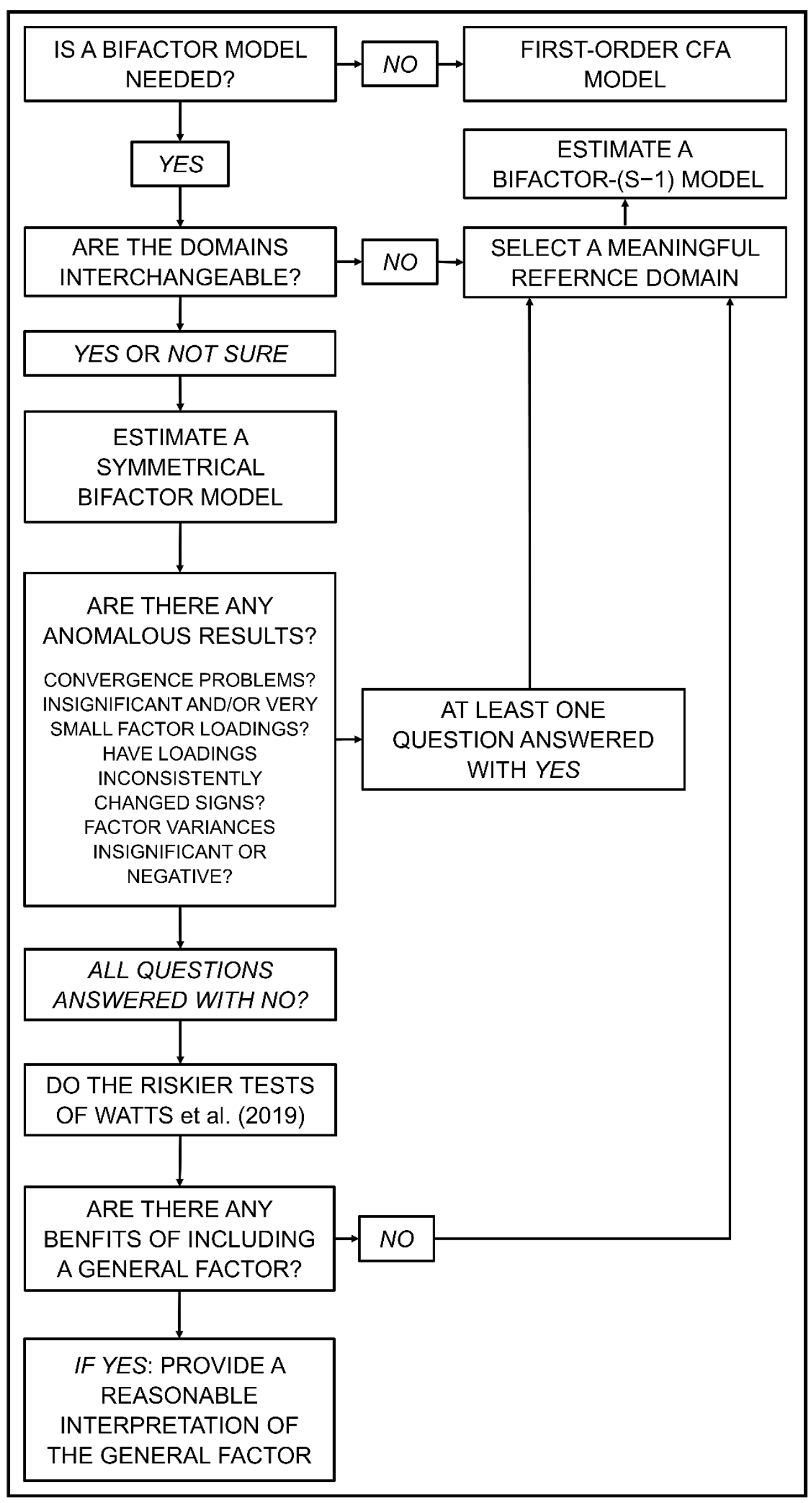

